\title{
Observational Manifestations of Young Neutron Stars: Spin-powered Pulsars
}

\author{
Michael Kramer \\ University of Manchester, Jodrell Bank Observatory, UK
}

\begin{abstract}
The largest number of known young neutron stars are observed as spin-powered pulsars. While the majority of those are detected at radio frequencies, an increasing number can be studied in other parts of the electromagnetic spectrum as well. The Crab pulsar is the prototype of a young pulsar which can be observed from radio to gamma-ray frequencies, providing a red thread of discussion during a tour through the pulsar properties observed across the electromagnetic spectrum. The basic observational features of pulsar emission are presented, preparing the ground for more detailed reviews given in these proceedings. Here, particular attention will be paid to those emission features which may provide a link between the radio and high-energy emission processes.
\end{abstract}

\section{Introduction}

It is an impossible task to summarize the diversity of pulsar phenomena observed across the electromagnetic spectrum on a few pages. The wealth of information that can be obtained outside the classical radio window today justifies some redefinition of the word "pulsar". Rather than being characterized by appearing as pulsating radio sources, a new definition of pulsar should be that it emits radiation that is pulsed due to rotation and powered by rotational energy. This definition also encompasses X-ray pulsars that are powered by the loss of rotational energy but which have not been detected at radio frequencies. This may be due to a misaligned radio beam or due to a very low radio luminosity. The discovery of an increasing number of very weak radio pulsars coincident with $\mathrm{X}$-ray point sources by Camilo and co-workers (see these proceedings) clearly shows that the expression "radio-quiet" cannot be used without the discussion of a corresponding flux density limit. Bearing this in mind, we will use "radioquiet" in the context of sources where pulsations have been detected at high energies but not (yet) at radio frequencies, assuming that there is no fundamental difference in the physics of these objects. Also, concentrating on young pulsars, we will neglect the whole population of millisecond pulsars which appear, however, to function under the same underlying principles as young pulsars (Kramer et al. 1998). There is not sufficient room to discuss these principles in a detailed theoretical framework, but observational implications for a working theory will be pointed out. Young, slowly-rotating pulsars known as "magnetars" (SGRs/AXPs) are discussed by Kaspi in these proceedings. 


\section{The Population of Young Pulsars}

The majority of spin-powered pulsars are still discovered and studied at radio frequencies. They dominate by far the number of about 1600 pulsars currently known, of which most are shown in the $P-\dot{P}$ diagram presented in Figure 1 . We will define "young pulsars" as those with a characteristic age, $\tau=P / 2 \dot{P}$, of less than $100 \mathrm{kyr}$. About 80 of these are currently known, and they are located in the upper left area of Figure 1. Specifically, pulsars with characteristic ages of less than $10 \mathrm{kyr}$ appear in the cross-hatched area, while pulsars with ages between 10 and $100 \mathrm{kyr}$ are located in the hatched area. The latter pulsars are often compared to the Vela pulsar if they match its spin-down luminosity, i.e. $\dot{E} \gtrsim 10^{36} \mathrm{ergs} \mathrm{s}^{-1}$. A corresponding line of constant $\dot{E}$ is shown together with a line for $\dot{E}=10^{33} \mathrm{ergs} \mathrm{s}^{-1}$. About 26 Vela-like pulsars are currently known (Kramer et al. 2003a). In comparison, there are only six Crab-like pulsars, including the fastest rotating young but radio-quiet pulsar PSR J0537-6910 with a period of only $16 \mathrm{~ms}$, the "Crab-twin" PSR B0540-69, PSR J1124-5916, and PSR B1509-58. In the same group we also find PSR J1119-6127 with the youngest age and highest $\dot{P}$ for any radio pulsar while the overall records are currently set by the radio-quiet PSR J1846-0258 in Kes 75 with a period of $323 \mathrm{~ms}$ and characteristic age of only $722 \mathrm{yr}$ (Gotthelf et al. 2000). This raises the question about the birth periods of pulsars which have currently been estimated for about nine sources (e.g. Migliazzo et al. 2002), covering one order of magnitude from less than $14 \mathrm{~ms}$ for PSR J0537-6910, over $19 \mathrm{~ms}$ for the Crab pulsar, to $140 \mathrm{~ms}$ for PSR J0538+2817 (Migliazzo et al. 2002; Kramer et al. 2003b). For some of these estimates, a measured braking index was needed. In total, five pulsars have a measured braking index that seems to reflect a physical braking process (rather than timing noise) with values ranging from $n=1.4$ to $n=2.9$ (e.g. Zhang et al. 2001).

\section{Radio Properties}

The radio properties of pulsars are discussed by Gupta (these proceedings). Therefore, we do not attempt to present or discuss the rich assortment of radio phenomena, but briefly review those which we consider to be important in the context of the bigger picture and their connection to high energy emission. In comparison to high energies, the energy output in the radio is tiny (see $\S 7$ ), with a median luminosity observed at $1400 \mathrm{MHz}$ of $L_{1400}=25 \pm 5 \mathrm{mJy} \mathrm{kpc}$. This number is biased by distant sources, so that $L_{1400}=3 \pm 1 \mathrm{mJy} \mathrm{kpc}^{2}$ computed for pulsars within $3 \mathrm{kpc}$ is a more representative number. There is a slight trend for older pulsars to be less luminous but this is mostly suggested by the millisecond pulsars which are indeed less luminous and less efficient radio emitters (Kramer et al. 1998). The flux density spectrum, $S \propto \nu^{\alpha}$, is steep with a mean spectral index of $\alpha \sim-1.7$. No age dependence is found despite earlier reports to the contrary (Maron et al. 2000). The highest radio frequency that pulsars have been detected at is $87 \mathrm{GHz}$ (Morris et al. 1997) and there are indications for a spectral turn-up at mm-wavelengths (Kramer et al. 1996). These observations are interesting due to a suggested "radius-to-frequency mapping" (RFM), according to which high frequency radio emission originates from 


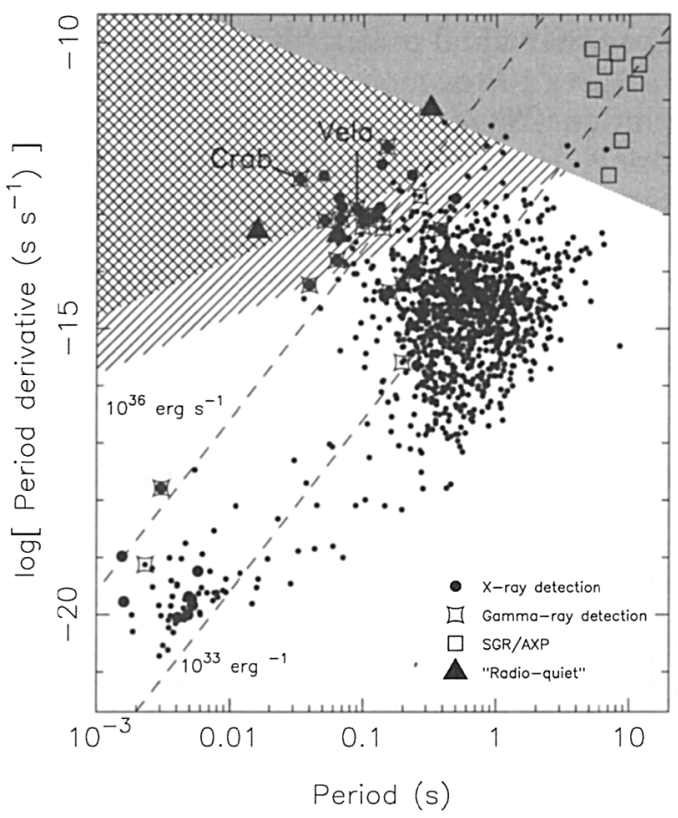

Figure 1. $\quad P-\dot{P}$ diagram of the currently known population of pulsars. The hatched area contains pulsars with spin-down ages of less than $100 \mathrm{kyr}$, while the cross-hatched area marks young pulsars with an age less than $10 \mathrm{kyr}$. Pulsars located in the grey area exhibit surface magnetic fields, $B_{S}=3.2 \times 10^{19} \sqrt{P \dot{P}}$ gauss, above the quantum critical field. Lines of constant spin-down luminosity of $\dot{E}=10^{33} \mathrm{ergs} \mathrm{s}^{-1}$ and $\dot{E}=10^{36}$ ergs s$^{-1}$ are shown. Pulsars marked as large filled circles have been detected at X-ray frequencies, and pulsars with $\gamma$-ray detections are shown by sharply-edged squares. Normal squares indicate neutron stars identified as SGRs or AXPs. Spin-powered pulsars with not (yet?) visible as normal radio pulsars are shown as filled triangles.

closer to the neutron star surface than low frequency emission (Cordes 1978). The model is motivated by the observation that pulse profiles become narrower at high frequencies. However, it seems difficult to decide whether the derived magnetospheric altitudes correspond to the heights where the coherent, usually highly polarized emission is created or where it escapes the magnetosphere after it had been created further down and propagated to the escape radius, possibly in certain wave modes (see contributions by Gupta, Petrova and Karastergiou et al.). In any case, there seems to be no doubt that the classical radio emission originates from a few hundred $\mathrm{km}$ or so above the pulsar surface (e.g. Kramer et al. 1997), and that the radiating plasma originates from the polar cap region. The interesting question is thereby how this plasma is related to that creating high energy emission, for which competing models place the origin near the polar cap as well (e.g. Daugherty \& Harding 1996) or further out in the magnetosphere in so-called "outer gaps" (e.g. Cheng, Ho \& Ruderman 1986). Clues are available from giant pulses observed for a handful of pulsars at radio frequen- 
cies. Giant pulses are individual pulses with flux densities exceeding the mean value by a factor of 10 to 100 or more. Recently published results for the Crab pulsar by Hankins et al. (2003) reveal fine-structure on nanosecond timescales with brightness temperatures reaching $10^{37} \mathrm{~K}$. Giant pulses tend to be aligned with the high energy pulses rather than with the radio profile (see §7). This suggests a common origin, indicating that some observed radio emission could be in fact a by-product of the high energy radiation process. This could explain the highly unusual "High-Frequency Components", seen to emerge at some odd pulse phases in the Crab profile at a few $\mathrm{GHz}$ (Moffett \& Hankins 1996), as the result of such possible by-products and geometrical RFM-like effects. This likely possibility underlines our need to take geometry into account if we want to understand both the observed radio as well as the high-energy emission.

\section{Optical Properties}

Currently, only five pulsars are reported to show pulsed optical emission (see contribution by Mignani or Shearer \& Golden 2002 for a review). The prime example is the Crab pulsar which is strong enough to allow extensive studies of its single pulses and their polarization properties, revealing interesting differences between the peak emission of the prominent double-peaked profile and the bridge and non-zero off-pulse emission (e.g. Romani et al. 2001; Kanbach et al. 2003). The similarity between the optical, X-ray and $\gamma$-ray profiles suggests that the emission is created by the same incoherent non-thermal radiation process, presumably at the same location. The polarization measurements and the application of the rotating-vector model (Radhakrishnan \& Cooke 1969) thereby allow us to study the geometry of the corresponding emission region in a way that is not yet possible in X-rays. Such studies are not possible for the other weaker pulsars with detected pulsed emission, i.e. Vela, Geminga and PSRs B0656+14 and B0540-69. Unpulsed emission has been detected for four pulsars, including PSR B1055-52, which together with the Crab, Vela, Geminga and possibly B0656+14 are also detected as $\gamma$-ray pulsars (e.g. Thompson 2001), again suggesting that the optical emission is closely related to X-ray and $\gamma$-ray emission. For a discussion of the luminosities involved it is important to note that for both PSRs B1055-52 and B0656+14 the most recent distance estimates dropped by a factor of $\sim 2$ (see Kramer et al. 2003a; Brisken et al. 2003).

\section{X-ray Properties}

On the one hand, X-ray observations promise to offer a more direct insight into the magnetospheric plasma densities and processes when compared to the view provided by radio observations that is somewhat occluded by the (unknown) physics responsible for the coherence of the radio emission. On the other hand, in many pulsars the observed X-ray emission is due to a mixture of thermal and non-thermal processes which cannot always be discriminated by the available data. Non-thermal, magnetospheric emission can be created by an accelerated relativistic plasma and should be highly pulsed with a power-law spectrum ranging from optical to $\gamma$-ray frequencies. Thermal emission may originate from the stellar surface and may still show low-amplitude modulation due to the rotating 
hot polar cap. In this case, we would expect a blackbody spectrum that is modified by a possible atmosphere of the neutron star, ranging from optical to soft $\mathrm{X}$-ray frequencies. In addition, matters may be complicated due to unresolved emission from pulsar-driven synchrotron nebulae or pulsar winds interacting with dense ambient media. Neglecting X-ray detections of millisecond pulsars, pulsed emission has been observed for 15 radio pulsars while nine pulsars have only been seen as unpulsed X-ray sources (Fig. 1; see Becker \& Aschenbach 2002 for a review). In addition, Geminga, the neutron star in PKS 1209-51/52, and PSRs J0537-6910, J1811-1925 and J1846-0258 are clearly spin-powered pulsars at X-rays but have no corresponding radio detection, yet. An account of recent deep searches in X-ray sources is given by Camilo (these proceedings), which led, for instance, to the discovery of radio pulses from the 66-ms pulsar J0205+6449 in SNR 3C 58. This pulsar would supersede the Crab as the youngest radio pulsar if $3 \mathrm{C} 58$ is indeed associated with the historical supernova SN 1181 (Murray et al. 2002). In general, it is instructive to group the X-raydetected pulsars into Crab-like sources, Vela-like sources and middle-aged pulsars. The Crab-like pulsars include PSRs B0540-69, J0537-6909, B1509-58, $\mathrm{J} 0205+6449$ and J1617-5055, all of which show strong features of magnetospheric emission and doubled-peaked profiles which are, if detected, aligned with similar optical profiles. For Vela-like pulsars, only Vela itself has been detected as a faint optical source. Their X-ray spectra do not represent simple powerlaws, while the pulse profiles are more complicated and typically misaligned with radio or $\gamma$-ray pulses. Finally, the middle-aged pulsars, i.e. Geminga, PSR B1055-52 and PSR B0656+14, show a mixture of thermal and non-thermal emission with energy-dependent profiles. The non-thermal emission is thought to originate either from the polar cap region (e.g. Daugherty \& Harding 1996) or outer gaps (e.g. Cheng et al. 1986; Romani 1996).

\section{6. $\gamma$-ray Properties}

The $\gamma$-ray emission of pulsars is highly pulsed, beamed and of non-thermal origin. There are seven "classical" $\gamma$-ray detections, i.e. Crab, Vela, Geminga and PSRs B1055+53, B1509-58, B1706-44 and B1951+32 (see e.g. Thompson 2001 or Kanbach 2002 for recent reviews). Their typically double-peaked profiles change their appearances towards higher energies due to a phase-dependent spectral hardness, suggesting, again, that geometry plays an important role. Similar studies are not possible for other, much weaker pulsars for which a detection has been suggested. Here, significance levels are much lower, and often their detection is based on the positional coincidence of a known radio pulsar with an unidentified $\gamma$-ray point source. A viable pulsar candidate should have properties consistent with those of the classical $\gamma$-ray pulsars, shown to be steady $\gamma$-ray sources (e.g. McLaughlin et al. 1996). While the beaming fraction is uncertain, the $\gamma$-ray luminosity seems to be a few per cent of $\dot{E}$. Until recently, this $\gamma$-ray efficiency was believed to reach much larger values, up to $20 \%$. However, this value was set by PSR B1055-52 for which the updated distance estimate (see $\S 4)$ now results in an efficiency of only $4 \%$. A recent detailed discussion of the proposed associations was presented by Kramer et al. (2003a) who estimated that about $19 \pm 6$ of the associations are genuine. Ultimately, instruments like 
$G L A S T$ will be needed to verify most of these candidates. By extending the observed $\gamma$-ray spectrum to a few tens of $\mathrm{GeV}, G L A S T$ may also be able to distinguish between the outer gap and polar cap model. The polar cap model interprets the observed emission as inverse Compton scattering of upward polar cap cascades and expects a cut-off of the spectrum due to $\gamma$ - $B$-field absorption. In contrast, the spectrum expected for the outer gap particles is curvatureradiation limited and should extend to higher energies (e.g. Thompson 2001).
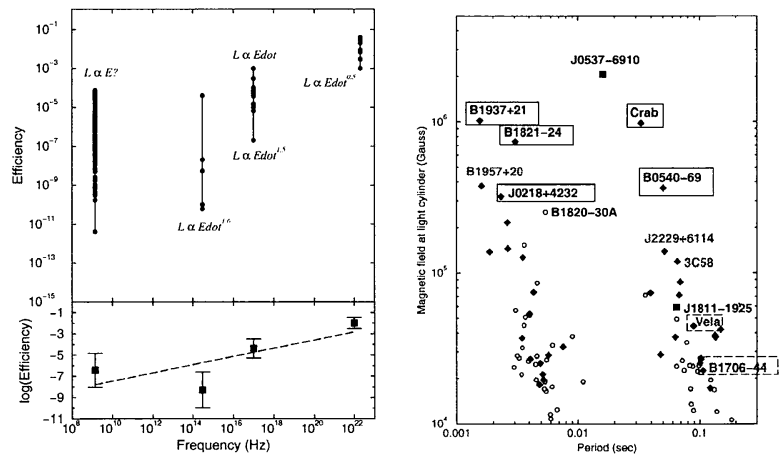

Figure 2. Left panel: Efficiency, $\eta \equiv L_{\nu} / \dot{E}$, as derived for radio, optical, X-ray and $\gamma$-ray frequencies. A fit to the median values shows an increase of efficiency with frequency of $\eta \propto \nu^{0.17 \pm 0.10}$. Right panel: Magnetic field strength at the light cylinder. Filled circles mark pulsars detected both at radio and high-energies. Radio-quiet pulsars are shown as filled squares. Pulsars with detected giant pulse emission are surrounded by a box. The Vela pulsar and PSR B1706-44 show so-called "micro-giants" (see Johnston \& Romani, these proceedings.)

\section{Relation to Spin Parameters}

It would be naive to assume that the radiation processes are governed by a single or a few control variables. It is nevertheless intriguing to relate some of the radiation properties to the spin-down luminosity and the magnetic fields at the surface and light cylinder.

A comparison of the energy output at radio, optical, X-ray and $\gamma$-ray frequencies is complicated by uncertain distance estimates, different spectral shapes, instrumental effects and in particular by the unknown beaming fractions. The radio emission appears to be emitted in a cone of radius $\rho$ that scales with period as $\rho \propto 1 / \sqrt{P}$ (e.g. Kramer et al. 1998). Due to the potential mixture of thermal and non-thermal emission observed, at optical and X-rays one typically assumes isotropic emission in the computation, even though this is obviously wrong for non-thermal emission. At $\gamma$-rays a beaming fraction of $1 / 4 \pi$ is adopted due to the lack of better knowledge. Bearing these uncertainties in mind, we can try to compare the derived luminosities "observed" at the different frequencies with the spin-down luminosity, $\dot{E}$. At radio no correlation can be found between $L_{\text {radio }}$ and $\dot{E}$. This is not unexpected given the importance of 
geometry and the complicated processes that must be present to create coherent emission. In the optical range, one can use peak luminosities to mitigate geometrical issues or luminosities integrated over the pulse profile (Shearer \& Golden 2002). Using the new distance for PSR B0656+14 has a significant im-

pact on the low number statistics, and we find $L_{\text {opt }} \propto \dot{E}^{1.6 \pm 0.2}$ (see Fig. 2). The X-ray luminosity, $L_{X}$, was studied by a number of authors, including Becker \& Trümper (1997) who found $L_{X} \propto E$ at soft X-rays $(0.1-2.4 \mathrm{keV})$. More recently, Possenti et al. (2002) reviewed the harder X-rays $(2-10 \mathrm{keV})$, deriving a relationship that is remarkably similar to that at optical frequencies, $L_{X} \propto \dot{E}^{1.5}$. At $\gamma$-rays, the low number statistics is again affected by revised distances for PSRs B1055-52 and J0218+4232 (Kramer et al. 2003a) but the values are consistent with $L_{\gamma} \propto \dot{E}^{0.5}$ (Thompson 2001). In conclusion, the inferred efficiencies, $\eta_{\nu}=L_{\nu} / \dot{E}$, show large variation at each frequency band, but as a general trend they increase from radio to $\gamma$-rays. A formal fit gives $\eta_{\nu} \propto \nu^{0.17 \pm 0.10}$ (Fig. 2).

In order to explain the lack of radio emission from magnetars (see Kaspi, these proceedings), it has been suggested that a surface magnetic field exceeding $B_{\text {crit }}=m_{e}^{2} c^{3} / e \hbar=4.4 \times 10^{13}$ gauss, would quench the radio emission (Zhang \& Harding 2000; see Fig. 1). However, the discoveries of pulsars with magnetar-like spin-parameters, like the 6-s PSR J1847-0130 with $B_{S} \sim 10^{14}$ gauss (McLaughlin et al. 2003), put into question the importance of the actual field value for the emission process. A more directly observable effect may be caused by the magnetic field at the light cylinder. It has been noted that pulsars with detected giant pulses happen to have the largest field strengths (Fig. 2; see contributions by Johnston and Joshi et al.) and that in general the giant pulse emission seems to align with the high energy emission. Indeed, there also appears to be a direct observational link between the giant radio pulses of the Crab and its optical emission (Shearer et al. 2003). Giant pulses may finally provide the clue to connect the emission across the whole electromagnetic spectrum.

\section{Summary and Conclusions}

Apparently, the radio emission originates from close to the stellar surface, while the high energy emission may tend to be created further out. In that picture, one would not expect an alignment of radio and high-energy emission, as typically observed. Where alignment is observed, like for the Crab pulsar, the observed radio emission may be of different origin and more related to that at high energies. Only the Crab's precursor component may be considered as the classical radio pulse, while the main and interpulse are by-products of high energy processes causing also the High-Frequency Components at a few $\mathrm{GHz}$. If that is the case, the Crab pulsar should be considered as a much less luminous radio source, similar to PSR B0540-69 when ignoring its giant pulse emission (Johnston \& Romani 2003). In summary, we have reason to believe that optical, X-ray and $\gamma$-ray processes are related and that they connect to the radio via giant pulses. A lot appears to be determined, or at least influenced, by geometry, and no single control parameter exists. There is still a lot to be done and understood.

Acknowledgments. I thank the RAS for a substantial travel grant. 


\section{References}

Becker W., \& Aschenbach, B. 2002, in MPE Rep. 278, Neutron Stars, Pulsars, and Supernova Remnants, eds. W. Becker, H. Lesch, \& J. Trümper, (Garching: MPE), p. 64

Becker, W., \& Trümper, J. 1997, A\&A, 326, 682

Brisken, W. F., et al. 2003, ApJ, 593, L89

Cheng, K. S., Ho C., \& Ruderman, M. 1986, ApJ, 300, 500

Cordes, J. M. 1978, ApJ, 222, 1006

Daugherty, J. K., \& Harding, A. K. 1996, ApJ, 458, 278

Gotthelf, E. V., et al. 2000, ApJ, 542, L37

Hankins, T. H., et al. 2003, Nature, 422, 141

Johnston, S., \& Romani, R. W. 2003, ApJ, 590, L95

Kanbach, G. 2002, in MPE Rep. 278, Neutron Stars, Pulsars, and Supernova Remnants, eds. W. Becker, H. Lesch, \& J. Trümper, (Garching: MPE), p. 91

Kanbach, G., et al. 2003, in Proc. of the SPIE, Instrument Design and Performance for Optical/Infrared Ground-based Telescopes, eds. I. Masanori, \& A. Moorwood, 4841 , p. 82

Kramer, M., et al. 1996, A\&A, 306, 867

- 1997, A\&A, 322, 846

- 1998, ApJ, 501, 270

- 2003a, MNRAS, 342, 1299

- 2003b, ApJ, 593, L31

Maron, O., et al. 2000, A\&AS, 147, 195

McLaughlin, M. A., et al. 1996, ApJ, 473, 763

- 2003, ApJ, 591, L135

Migliazzo, J. M., et al. 2002, ApJ, 567, L141

Moffett, D. A., \& Hankins, T. H. 1996, ApJ, 468, 779

Morris, D., et al. 1997, A\&A, 322, L17

Murray, S. S., et al. 2002, ApJ, 568, 226

Possenti, A., et al. 2002, A\&A, 387, 993

Radhakrishnan, V., \& Cooke, D. J. 1969, Astrophys. Lett., 3, 225

Romani, R. W. 1996, ApJ, 470, 469

Romani, R. W., et al. 2001, ApJ, 563, 221

Shearer, A., \& Golden, A. 2002, in MPE Rep. 278, Neutron Stars, Pulsars, and Supernova Remnants, eds. W. Becker, H. Lesch, \& J. Trümper, (Garching: MPE), p. 44

Shearer, A., et al. 2003, 301, 493

Thompson, D. J. 2001, in AIP Conf. Proc., Vol. 558, High Energy Gamma-Ray Astronomy, eds. F. A. Aharonian, \& H. J. Völk, (New York: AIP), p. 103

Zhang, B., \& Harding, A. K. 2000, ApJ, 535, L51

Zhang, W., et al. 2001, ApJ, 554, L177 